\title{
Adjuvant acupuncture reduces first trimester pregnancy loss after IVF
}

\author{
Naseem M. Khorram ${ }^{1}$, Susan Horton ${ }^{2}$, Vicken Sahakian², Richard Chacon ${ }^{2}$, Omid Khorram ${ }^{\text {* }}$ \\ ${ }^{1}$ Department of Obstetrics and Gynecology, Harbor-UCLA Medical Center, Torrance, USA \\ ${ }^{2}$ Department of Obstetrics and Gynecology, Pacific Fertility Center, Westwood, USA \\ Email: *okhorram@obgyn.humc.edu
}

Received 25 April 2012; revised 30 May 2012; accepted 11 June 2012

\begin{abstract}
Background: The objective of this study was to determine the effects of acupuncture treatment as an adjunctive therapy on the outcome of in vitro fertilization (IVF)/Intracytoplasmic Sperm Injection (ICSI). Material/Methods: We conducted a retrospective study of 238 patients who underwent conventional IVF/ ICSI alone or in conjunction with acupuncture over a course of 2 years. Patients in the two treatment groups were matched in terms of age and diagnosis. Acupuncture was administered in two sessions 5 to 7 days prior to and on the day of embryo transfer. Results: There were no differences between the two groups in terms of fertilization rate, pregnancy or implantation rates, and endometrial thickness. The number of oocytes retrieved $(P<0.005)$ and the number of first trimester miscarriages were significantly lower in the group receiving acupuncture $(P<$ 0.001). Conclusions: Acupuncture reduces miscarriage rates in patients undergoing IVF/ICSI possibly secondary to stress relief.
\end{abstract}

Keywords: Acupuncture; IVF/ICSI; Pregnancy Rates; Implantation Rate; Miscarriage

\section{INTRODUCTION}

Acupuncture, an ancient Chinese treatment, is based on the energy flow of Qi. Disease results when this energy flow is disrupted, and can subsequently be treated via stimulation of specific points on the body that balance this energy flow [1]. In recent studies, the use of acupuncture in conjunction with in vitro fertilization (IVF) treatments has produced mixed results with both positive and no effects. In relation to female infertility, acupuncture is believed to stimulate $\beta$-endorphin secretion which in turn stimulates blood flow to the uterus and ovaries [2]. The principle mechanisms by which acupuncture could

*Corresponding author. improve the outcome of IVF are thought to be: modulation of neuroendocrine factors; increased blood flow to the uterus and ovaries; cytokine modulation; and reduction of stress and anxiety [3].

In a randomized controlled study Paulus et al. [4] reported a higher pregnancy rate in women who received acupuncture both before and after embryo transfer was reported. This study was limited due to lack of a placebo control group $[4,5]$. In a retrospective study by Magarelli et al. [6] higher pregnancy rates $(51 \%$ vs $36 \%, \mathrm{P}<0.05)$ as well as lower miscarriage rates $(8 \%$ vs $20 \%, \mathrm{P}<0.05)$ among poor responder women who received acupuncture was reported. In a subsequent study by the same investigators significantly higher pregnancy rates for good prognosis patients receiving acupuncture (53\% vs $38 \%$, $\mathrm{P}<0.01)$ was reported [7]. However, in a study conducted by Wang et al. [8] where acupuncture treatments were only performed during the follicular and luteal phase but not on the day of embryo transfer no difference in pregnancy rates was found. The aim of this study was to determine the effects of acupuncture treatment delivered by a single acupuncturist as an adjunctive therapy on the outcome of IVF/ICSI and the resultant pregnancies in two groups of women matched for age and diagnosis.

\section{MATERIALS AND METHODS}

This study was a retrospective analysis using data collected at Pacific Fertility Center over a two year course. Data was obtained from 127 patients having IVF/ICSI in conjunction with acupuncture, and another 111 patients undergoing IVF/ICSI alone. Being a retrospective chart analysis the study was considered as IRB exempt. Patients in the study were derived from the practices of two physicians working in the same center and using the same embryology laboratory with one physician referring all their patients for acupuncture and the other physician not referring their patients for acupuncture. Both physicians are board certified reproductive endocrinolo- 
gists using the same stimulation protocols and medications for controlled ovarian hyperstimulation. The stimulation protocol consisted of oral contraceptive suppression for one month followed by Leuprolide acetate for down regulation. For controlled ovarian hyperstimulation a combination of recombinant FSH and HMG preparation (1:1 or $2: 1$ ratio) was used. Luteal support was provided with progesterone in oil $(50 \mathrm{mg} / \mathrm{ml} \mathrm{IM}$, daily) and vaginal suppository (50 mg daily). A single acupuncturist administered the treatment. A minimum of two acupuncture treatments were given 5 to 7 days prior to and on the day of embryo transfer. The acupuncture protocol consisted of three groups of acupuncture points, each group promoting fertility in a different way. These groups were Eight Extraordinary Vessel points, Twelve Meridian acupuncture points, and Auricular points. Additionally, each session incorporated acu-points selected to treat specific presenting symptoms related to side effects of the fertility medications.

The first group of points inserted pertains to the deep lying Extraordinary Vessels. The combination of Pericarium Six and Spleen Four opens the Chong Mai, the Sea of Blood, which promotes blood flow to the uterus. The combination points Lung 7 and Kidney 6 open the Ren Mai, the Receiving Vessel, tonifying all aspects of reproduction and fertility. The second group of points is related to the meridians located more superficially on the body. Stomach 36, Spleen 6, Kidney 3, Ren 4 (conception vessel 4), Stomach 28, Liver 3 and Large Intestine 4 all work together to activate and strengthen Qi and Blood.

The data were then analyzed documenting seven variables including the clinical outcome, as well as age, diagnosis, endometrial thickness, number of oocytes retrieved, percent fertilization, and implantation rates. The implantation rate was calculated by dividing the number of gestational sacs on the ultrasound by the number of embryos transferred. All pregnancies were followed for the first trimester. Data was analyzed using the Sigma Stat software. The Chi-square and student's t-test were used to compare the various end points in the two study groups.

\section{RESULTS}

Table 1 demonstrates the demographics of the two study groups. As shown there were no differences in terms of age or infertility diagnosis or percentage of patients undergoing Intracytoplasmic Sperm Injection (ICSI) between the two groups. Outcome data are shown in Table 2. There were no significant differences between the groups in terms of endometrial thickness, percent fertilization, or pregnancy rates. The number of oocytes retrieved in the control group was significantly higher
Table 1. Diagnosis and age of patients in acupuncture and control groups.

\begin{tabular}{cccc}
\hline & $\begin{array}{c}\text { Control } \\
(\mathbf{n}=\mathbf{1 1 1})\end{array}$ & $\begin{array}{c}\text { Acupuncture } \\
(\mathbf{n = 1 2 7})\end{array}$ & $\mathbf{P}$ \\
\hline Average Age & $36.61 \pm 0.41$ & $36.83 \pm 0.51$ & $\mathrm{NS}$ \\
Ovulatory Dysfunction & $8.3 \%$ & $11 \%$ & $\mathrm{NS}$ \\
Male Factor & $33 \%$ & $38.5 \%$ & $\mathrm{NS}$ \\
Tubal Disease & $13 \%$ & $9.7 \%$ & $\mathrm{NS}$ \\
Unexplained & $16.2 \%$ & $20 \%$ & $\mathrm{NS}$ \\
Age Factor & $8.3 \%$ & $5.3 \%$ & $\mathrm{NS}$ \\
Endometriosis & $21 \%$ & $15.8 \%$ & $\mathrm{NS}$ \\
\hline
\end{tabular}

Table 2. End points analyzed in acupuncture and control groups.

\begin{tabular}{cccc}
\hline & $\begin{array}{c}\text { Control } \\
(\mathbf{n}=\mathbf{1 1 1})\end{array}$ & $\begin{array}{c}\text { Acupuncture } \\
(\mathbf{n}=\mathbf{1 2 7})\end{array}$ & $\mathbf{P}$ \\
\hline $\begin{array}{c}\text { Endometrial Thickness } \\
(\mathbf{m m})\end{array}$ & $8.73 \pm 0.21$ & $8.29 \pm 0.19$ & $\mathrm{NS}$ \\
$\begin{array}{c}\text { Mean Number of } \\
\text { Oocytes Retrieved }\end{array}$ & $13.55 \pm 0.88$ & $10.57 \pm 0.60$ & 0.005 \\
Percent Fertilization & $66.36 \% \pm 2.09 \%$ & $68.97 \% \pm 2.29 \%$ & $\mathrm{NS}$ \\
Implantation Rate & $34.74 \% \pm 3.29 \%$ & $31.12 \% \pm 3.28 \%$ & $\mathrm{NS}$ \\
Pregnancy Rate & $45.45 \% \pm 0.04 \%$ & $39.37 \% \pm 0.04 \%$ & $\mathrm{NS}$ \\
Miscarriage Rate & $40 \% \pm 0.07 \%$ & $31.8 \% \pm 0.07 \%$ & 0.001 \\
\hline
\end{tabular}

than acupuncture group $(\mathrm{P}<0.005)$. First trimester miscarriage rates were significantly lower $(\mathrm{P}<0.001)$ in the acupuncture group as compared with the control group. The $95 \%$ confidence interval for the difference between the two means (miscarriage) was determined to be -0.280 to +0.117 making it plausible that $\mu_{1}$ and $\mu_{2}$ are nearly equal and the null hypothesis can be accepted.

\section{DISCUSSION}

In this study comparing two well-matched groups undergoing IVF/ICSI alone or in conjunction with acupuncture no differences in terms of endometrial thickness, fertilization, or pregnancy rates was observed. However, the rates of first trimester miscarriage rates were significantly lower in the acupuncture group.

Patients undergoing IVF are often times under great emotional stress, and it has been found that stress can reduce fertility [9], and increase miscarriages [10]. Acupuncture can help eliminate mental stress $[11,12]$ even in the setting of patients undergoing IVF [13]. In our study subjects receiving acupuncture reported feeling relaxed after the treatment sessions, possibly indicating reduced stress in this group. The reduction in stress induced by acupuncture has been associated with higher $\beta$ - 
endorphin secretion [14]. At the time of embryo transfer uterine contraction frequency is increased [15], and acupuncture has been shown to reduce uterine contractions [16] and thereby increase pregnancy rates [17]. The reduction of uterine contractions might be another potential mechanism for reduction of miscarriage rates found in our study. In a systematic online review and meta-analysis of seven trials involving 1366 women undergoing IVF and IVF in conjunction with acupuncture treatments on day of embryo transfer [18] an increase in clinical pregnancy (odds ratio 1.65, 95\% confidence interval 1.27 to 2.14; number needed to treat (NNT) 10 (7 to 17); seven trials), ongoing pregnancy $(1.87,1.40$ to 2.49 ; NNT 9 (6 to 15$)$; five trials), and live birth (1.91, 1.39 to 2.64; NNT 9 (6 to 17); four trials) was reported. While the miscarriage rates were not specified, live birth rates were determined to be higher in the acupuncture group. Stener et al. [19] also reported a higher take home baby rate per embryo transfer in the acupuncture group (41\% versus $19.4 \%, \mathrm{P}<0.05)$. The miscarriage rate in this study was determined to be non-significantly lower in the acupuncture group, (10.7\% vs $31.6 \%)$. Magarelli et al. [20] found statistically lower ectopic pregnancy rates in the acupuncture group $(\mathrm{P} \leq 0.05)$ with no differences in implantation and fertilization rates or number of oocytes retrieved. Stener-Victorin et al. [19] also showed a significantly higher implantation rate per embryo transfer $(27.2 \%$ vs $16.3 \%, \mathrm{P}<0.05)$ in the acupuncture group. The reason for the lower number of oocytes retrieved in the acupuncture group in our study is unknown. If acupuncture increases blood flow to the ovary we would have expected to retrieve higher number of oocytes in the acupuncture group. The reported differences in outcomes in the various acupuncture studies are related to heterogeneity of the patients studied, the timing and manner of acupuncture administered. To control for these variables multi-center randomized prospective trials using the same protocols will be needed to address the beneficial or lack of effect of acupuncture as an adjunct to IVF/ICSI. The effects of acupuncture on uterine blood flow, contractions and correlation with stress level will need to be assessed in such trials.

\section{ACKNOWLEDGEMENTS}

The authors thank Ms. Hye Jin Park for manuscript preparation.

\section{REFERENCES}

[1] Dieterle, S., Ying, G., Hatzmann, W. and Neuer, A. (2006) Effect of acupuncture on the outcome of in vitro fertilization and intracytoplasmic sperm injection: A randomized, prospective, controlled clinical study. Fertility Sterility, 85, 1347-1351. doi:10.1016/j.fertnstert.2006.01.008

[2] Westergaard, L.G., Mao, Q., Krogslund, M., Sandrini, S.,
Lenz, S. and Grinsted, J. (2006) Acupuncture on the day of embryo transfer significantly improves the reproductive outcome of infertile women: A prospective, randomized trial. Fertility and Sterility, 85, 1341-1346. doi:10.1016/j.fertnstert.2005.08.070

[3] Anderson, B.J., Haimovici, F., Ginsburg, E.S., Schust, D.J. and Wayne, P.M. (2007) In vitro fertilization and acupuncture: Clinical efficacy and mechanistic basis. Alternative Therapeutic Health Medicine, 13, 38-48.

[4] Paulus, W.E., Zhang, M., Strehler, E., El-Danasouri, I. and Sterzik, K. (2002) Influence of acupuncture on the pregnancy rate in patients who undergo assisted reproduction therapy. Fertility and Sterility, 77, 721-724. doi:10.1016/S0015-0282(01)03273-3

[5] White, A.R. (2003) A review of controlled trials of acupuncture for women's reproductive health care. Journal of Family Planning and Reproductive Health Care, 29, 233-236. doi:10.1783/147118903101197863

[6] Magarelli, P.C. and Cridenda, D.K. (2004) Acupuncture and IVF poor responders a cure? Fertility and Sterility, 81, S20. doi:10.1016/j.fertnstert.2004.02.045

[7] Magarelli, P.C., Cridenda, D.K. and Cohen, M. (2004) Acupuncture and good prognosis IVF patients: Synergy. Fertility and Sterility, 82, S80-S81. doi:10.1016/j.fertnstert.2004.07.205

[8] Wang, W., Check, J.H., Liss, J.R. and Choe, J.K. (2007) A matched, controlled study to evaluate the efficacy of acupuncture for improving pregnancy rates following in vitro fertilization-embryo transfer. Clinics Experimental Obstetetrics \& Gynecology, 34, 137-138.

[9] Csemiczky, G., Landgren, B.M. and Collins, A. (2000) The influence of stress and state anxiety on the outcome of IVF-treatment: Psychological and endocrinological assessment of Swedish women entering IVF-treatment. Acta Obstetrics \& Gynecology Scandinivia, 79, 113-118. doi:10.1034/j.1600-0412.2000.079002113.x

[10] Repromed (2007) Pregnancy rates. http://www.repromed.com.au/question5.html

[11] Middlekauff, H.R. (2004) Acupuncture in the treatment of heart failure. Cardiology Review, 12, 171-173. doi:10.1097/01.crd.0000103650.71735.f0

[12] Ernst, E. and White, A.R. (2001) Prospective studies of the safety of acupuncture: A systematic review. American Journal of Medicine, 110, 481-485. doi:10.1016/S0002-9343(01)00651-9

[13] Chan, C.H., Ng, E.H., Chan, C.L., Ho and Chan, T.H. (2006) Effectiveness of psychosocial group intervention for reducing anxiety in women undergoing in vitro fertilization: A randomized controlled study. Fertility and Sterility, 85, 339-346. doi:10.1016/j.fertnstert.2005.07.1310

[14] Dong, J.T. (1993) Research on the reduction of anxiety and depression with acupuncture. American Journal of Acupuncture, 21, 327-330.

[15] Ayoubi, J.M., Epiney, M., Brioschi, P.A., Fanchin, R., Chardonnens, D. and de Ziegler, D. (2003) Comparison of changes in uterine contraction frequency after ovulation in the menstrual cycle and in in vitro fertilization cycles. Fertility and Sterility, 79, 1101-1105. 
doi:10.1016/S0015-0282(03)00179-1

[16] Kim, J., Shin, K.H. and Na, C.S. (2000) Effect of Acupuncture treatment on uterine motility and cyclooxygenase-2 expression in pregnant rats. Gynecologic and Obstetric Investigation, 50, 225-230. doi:10.1159/000010321

[17] Franchin, R., Righini, C., Olivennes, F., Taylor, S., de Ziegler, D. and Frydman, R. (1998) Uterine contractions at the time of embryo transfer alter pregnancy rates after in vitro fertilization. Human Reproduction, 13, 1968-1974. doi:10.1093/humrep/13.7.1968

[18] Manheimer, E., Zhang, G., Udoff, L., Haramati, A., Langenberg, P., Berman, B.M., and Bouter, L.M. (2008) Effects of acupuncture on rates of pregnancy and live birth among women undergoing in vitro fertilization: Systematic review and meta-analysis. British Medical Journal, 336, 545-549. doi:10.1136/bmj.39471.430451.BE

[19] Stener-Victorin, E., Waldenström, U., Nilsson, L., Wikland, M. and Janson, P.O. (1999) A prospective randomized study of electro-acupuncture versus alfentanil as anaesthesia during oocyte aspiration in in vitro fertilization. Human Reproduction, 14, 2480-2484. doi:10.1093/humrep/14.10.2480

[20] Magarelli, P.C., Cridennda, D. and Cohen, M. (2005) Improvement of IVF outcomes by acupuncture: Are egg and embryo qualities involved? Fertility and Sterility, 83, S9. 